\title{
Clues on Galaxy and Cluster Formation from their Scaling Relations
}

\author{
Barbara Lanzoni ${ }^{1}$, Luca Ciotti ${ }^{2}$, Alberto Cappi ${ }^{1}$, Giuseppe Tormen ${ }^{3}$, and \\ Gianni Zamorani ${ }^{1}$ \\ 1 INAF - Osservatorio Astronomico di Bologna, via Ranzani 1, 40127, Bologna, Italy \\ 2 Dip. di Astronomia, Università di Bologna, via Ranzani 1, 40127 Bologna, Italy \\ 3 Dip. di Astronomia, Università di Padova, vicolo dell'Osservatorio 5, 35122 Padova, \\ Italy
}

\begin{abstract}
By means of high-resolution N-body simulations in a $\Lambda$ CDM cosmology, we verify that scaling relations similar to those observed for nearby galaxy clusters are also defined by their dark matter hosts; the slopes, however, are not the same. We then show that the scaling relations of galaxy clusters can be explained as the result of the cosmological collapse of density fluctuations at the appropriate scales, plus a systematic trend of the $M / L$ ratio with cluster mass. The empirical fact that the exponent of the Faber-Jackson relation of elliptical galaxies is significantly different (higher) than that of clusters, force us to conclude that the galaxy scaling laws might derive from the cosmological collapse of density fluctuations at the epoch when galactic scales became non-linear, plus modifications afterward due to early-time dissipative merging.
\end{abstract}

\section{Observed and simulated scaling relations}

It has been shown $[7,1]$ that nearby galaxy clusters define scaling relations involving their optical luminosity $L$, effective radius $R_{\mathrm{e}}$ and velocity dispersion $\sigma$. Reanalyzing the sample of [7], we obtain $L \propto \sigma^{2.18 \pm 0.52}$ for the Faber-Jackson (FJ) relation, $L \propto R_{\mathrm{e}}^{1.55 \pm 0.19}$ for the Kormendy relation, and $L \propto R_{\mathrm{e}}^{0.9 \pm 0.15} \sigma^{1.31 \pm 0.22}$ for a nearly edge-on view of the Fundamental Plane, where $L$ is in $10^{12} L_{\odot} / h^{2}$, $\sigma$ in $1000 \mathrm{~km} / \mathrm{s}, R_{\mathrm{e}}$ in $\mathrm{Mpc} / h$.

Similar scaling laws are also followed by early-type galaxies, with the only significant difference of the FJ relation: in fact, $L \propto \sigma^{4}$ for luminous ellipticals [2].

For a sample of 13 massive $\left(M>10^{14} \mathrm{M}_{\odot} / h\right)$ DM halos obtained from Nbody cosmological simulations [4], we have computed the projected radius containing half the total mass $\left(R_{\mathrm{h}}\right)$ and the mean projected velocity dispersion within it $\left(\sigma_{\mathrm{h}}\right)$. Well defined scaling relations relating $R_{\mathrm{h}}, \sigma_{\mathrm{h}}$ and the total mass $M$ are found, and are interpreted as the result of the cosmological collapse of density fluctuations and the weak homology of DM halos [6]. The slopes of these relations, however, do not coincide with the observed ones: the simplest way to satisfy at once the theoretical and observational scaling laws is to assume that $M / L \propto L^{0.3}$, in good agreement with what inferred observationally [3].

Since a similar trend of $M / L$ with $L$ is also found for early-type galaxies, and the cosmological predictions for the DM halo scaling relations are the same 


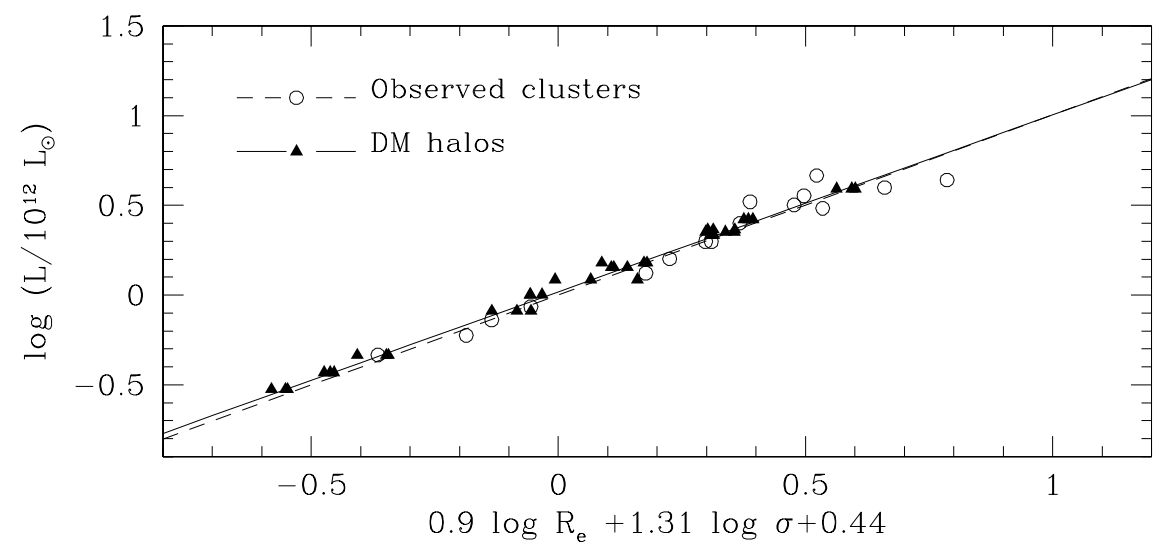

Fig. 1. Nearly edge-on view of the FP of galaxy clusters, and of their DM halos under the assumption $M / L \propto L^{0.3}$

at galactic and cluster scales, the higher slope of the galactic FJ relation implies that some additional mechanism played a role in the formation/evolution of ellipticals: indeed, the combined effect of dissipationless merging (which tends to increase the FJ exponent) and gas dissipation (which acts in the opposite direction) might be the solution [5].

\section{Conclusions}

We conclude that, while the scaling relations of galaxy clusters can be explained by the cosmological collapse of density fluctuations at the appropriate scale, plus a systematic trend the total $M / L$ ratio with cluster luminosity, the scaling laws of elliptical galaxies seem to suggest that additional processes (like early-time dissipational merging) probably played an important role after the cosmological collapse of the density fluctuations at these scales. For a discussion of how $M / L$ for clusters could increase with $L$, we refer to [5].

\section{References}

1. Adami C., Mazure A., Biviano A., Katgert P., Rhee G.: 1998a, A\&A 331, 493

2. Bernardi M., et al.: 2003a, AJ 125, 1882

3. Girardi M., Manzato P., Mezzetti M., Giuricin G., Limboz F.: 2002, ApJ 569, 720

4. Lanzoni B., Cappi A., Ciotti L.: Mem. S.A.It. Suppl. Vol. 1, 145

5. Lanzoni B., Ciotti L., Cappi A., Tormen G., Zamorani G.: ApJ in press (astroph/0307141)

6. Navarro J.F., Frenk C.S., White S.D.M.: 1997, ApJ 490, 493 (NFW)

7. Schaeffer R., Maurogordato S., Cappi A., Bernardeau F.: 1993, MNRAS 263, L21 